\title{
A Model of Hippocampal Competition between New Learning and Memory Updating
}

\author{
Antoine Besnard \\ Université Pierre et Marie Curie Paris 06-CNRS-UMR 7224, INSERM-UMRS952, F-75005 Paris, France \\ Review of Winters et al.
}

Memory consolidation is a time-dependent stabilization process that leads to permanent storage of newly acquired memory (McGaugh, 1966). However, compelling evidence has suggested that upon recall, memories can reenter states of transient instability (Nader and Hardt, 2009). This reconsolidation process was firmly established by the demonstration that a previously consolidated auditory fear memory in which a tone and electric footshock were associated could be impaired by microinjecting anisomycin (a protein synthesis inhibitor) into the basolateral nucleus of the amygdala immediately after reexposure to the tone (Nader et al., 2000). Much evidence now exists to support the hypothesis that reconsolidation may offer unique opportunities to update memories (Lee, 2009).

Recently, the reconsolidation hypothesis has been extended to memories that do not rely on any explicit reinforcement, such as object recognition memory (Davis et al., 2010). The object recognition task measures memory in rodents by exploiting their natural preference for interacting with novel rather than familiar objects (Ennaceur and Delacour, 1988). This task usually takes place in an open-field arena

\footnotetext{
Received Dec. 21, 2011; revised Jan. 24, 2012; accepted Jan. 24, 2012

This work was supported by the Centre National de la Recherche Scientifique. I thank Dr. Serge Laroche and Emma Cahill for their valuable comments on the text.

Antoine Besnard declares no financial conflicts of interest.

Correspondence should be addressed to Dr. Antoine Besnard, Université

Pierre et Marie Curie Paris 06-CNRS-UMR 7224, INSERM-UMRS952, 9 quai Saint Bernard75005 Paris, France.E-mail: antoine.besnard@snv.jussieu.fr. DOI:10.1523/JNEUROSCI.6368-11.2012

Copyright $\odot 2012$ the authors $\quad 0270-6474 / 12 / 323281-03 \$ 15.00 / 0$
}

where animals are exposed briefly to two or three objects. A memory test is conducted after a variable retention delay by exchanging one of the familiar objects for a novel object. Preferential exploration of the novel object provides an index of recognition memory.

In a recent paper published in The Journal of Neuroscience, Winters and colleagues (2011) used a modified version of the object recognition paradigm in which two identical objects were presented in a Y-shaped apparatus, one at the end of each exploration arm. The authors previously reported that the perirhinal cortex (PRh), but not the hippocampus (HPC), is necessary for object recognition in this version of the task, which minimizes the influence of spatial or contextual information (Winters et al., 2004).

In their follow-up study, Winters and colleagues (2011) infused the proteinsynthesis inhibitor anisomycin, which interferes with both consolidation and reconsolidation (Nader and Hardt, 2009), into the PRh or HPC immediately following memory reactivation. Although the authors addressed the role of both structures in the reconsolidation of object memory, here we will focus on the HPC contribution to object memory updating.

An important finding of the Winters and colleagues (2011) study is that following a reactivation session conducted in the presence of a novel object, anisomycin infusion into the HPC did not impair discrimination between novel and familiar objects on the subsequent day. The authors concluded that HPC protein synthesis inhibition upon recall did not alter the previously established object memory (Winters et al., 2011; their Fig. 5). These results are at odds with previous work by Rossato and colleagues (2007), in which anisomycin infusion into the HPC under the same circumstances prevented discrimination between novel and familiar objects. In this case, the authors concluded that HPC protein synthesis inhibition upon recall altered the previously established object memory. As discussed by Winters and colleagues (2011), this discrepancy may result from procedural differences: they emphasize the different contribution of HPC in the two paradigms and suggest that linking a new object to the original memory requires HPC function only in HPC-dependent tasks (as used by Rossato et al., 2007), not in HPC-independent tasks (as used by Winters et al., 2011). Although this is a rational explanation for the conflicting results, one must not forget an important caveat when considering memory updating: situations offering updating opportunities, which engage reconsolidation processes, may also lead to new learning, which involves consolidation processes independent of the original memory (Lee, 2009).

To incorporate additional features into the original mnemonic trace, memories must be reactivated (Lee, 2009). In this framework, procedural differences such as the degree of similarity between the events present at memory recall and the previously memorized experience may lead to opposite 
Original memory strength or age

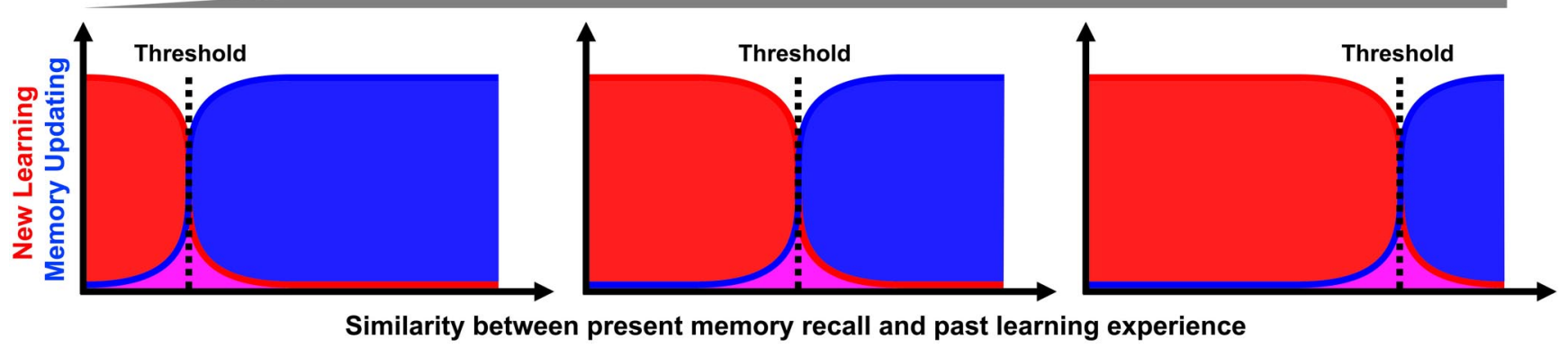

Figure 1. Schematic representation of the model of hippocampal competition between new learning and memory updating. Upon memory reactivation, the degree of similarity between the events present at recall and past learning experience may lead to opposite mnemonic processes. A low degree of similarity should prompt new learning (red curve), whereas the opposite should trigger updating of the original memory (blue curve). A threshold of similarity (dotted line) may gate the switch between new learning and memory updating. Some intrinsic properties of the original memory, such as the strength or the age of the memory, may also influence this threshold (from left to right). In the case of weakly or recently encoded memories, the low similarity threshold should favor the updating of the original memory upon reactivation (left). This threshold should become increasingly high in the case of strongly or remotely encoded memories, thus limiting the updating process to avoid catastrophic interference between already established memories and learning of independent information (right).

mutually exclusive mnemonic processes. I propose that a low degree of similarity should prompt new learning (Fig. 1, red curve), whereas a high degree of similarity should trigger updating of the original memory (Fig. 1, blue curve). Thus, a threshold of similarity may gate the switch between new learning and memory updating (Fig. 1, dotted lines). In addition, some properties of the original memory, such as the strength or the age of the memory (Lee, 2009), may also influence this threshold (Fig. 1, from left to right). Specifically, for weakly or recently encoded memories, the low similarity threshold should favor updating of the original memory upon reactivation (Fig. 1, left). This threshold should become increasingly high for strongly or remotely encoded memories, thus limiting the updating process to avoid catastrophic interference between already established memories and learning of independent information (Fig. 1, right).

In the work of Winters and colleagues (2011), because the Y-shaped apparatus minimizes the influence of spatial or contextual information, the original memory trace relies on the objects per se. Therefore, when memory reactivation was conducted in the presence of a novel object in the same context (Winters et al., 2011; their Fig. 5), the reactivation session was highly dissimilar to the sample session. In such cases, one cannot ensure that the original memory was reactivated. Anisomycin infusion into the HPC shortly after recall did not prevent discrimination between novel and familiar objects on the subsequent day, suggesting it spared the original object memory. Thus, the low degree of similarity may have prompted new learning instead of memory updating, as predicted by the current model (Fig. 1, red curve). In line with this interpretation, anisomycin infusion into the PRh prevented discrimination between the novel and familiar objects on subsequent day (Winters et al., 2011; their Fig. 4). These data are in accordance with a new learning process, because object memory acquisition depends on PRh but not HPC in this paradigm (Winters et al., 2004).

Conversely, when memory reactivation was conducted in the presence of a contextual change (Winters et al., 2011; their Fig. 7), the reactivation session was highly similar to the sample session, because the two familiar objects were presented in a slightly modified apparatus. Anisomycin infusion into the HPC shortly after recall impaired the original object memory. In this case, the higher degree of similarity may have encouraged updating of the original memory, as predicted by the current model (Fig. 1, blue curve).

This alternative proposal also reconciles these results with previous findings obtained in an open-field arena (Bozon et al., 2003; Rossato et al., 2007). In these studies, the original memory involved objects belonging to a far more complex environment and reconsolidation occurred only when reactivation involved the two familiar objects in the training context, not when they were presented in a different context (Bozon et al., 2003). In the latter case, the reactivation session was highly dissimilar to the sample session because only the two familiar objects from the complex environment were present. Thus, the low degree of similarity may have encouraged new learning instead of memory updating, as predicted by the current model (Fig. 1, red curve).

In the work of Rossato and colleagues (2007), when memory reactivation was conducted in the presence of a novel object, the reactivation session was highly similar to the sample session because only one object from the complex environment was changed. Anisomycin infusion into the HPC shortly after recall impaired the original object memory, as indicated by the failure to discriminate between novel and familiar objects on the subsequent day. In this case, the high degree of similarity may have resulted in updating of the original memory, as predicted by the current model (Fig. 1, blue curve).

The present model may also apply to fear-related memories. Indeed, linking new information to a reactivated memory has often been reported to engage consolidation (Tronel et al., 2005) or reconsolidation processes in the HPC (Lee, 2010). In the work of Tronel and colleagues (2005), rats learned to associate an electric footshock with a light in context A. In this situation, the robust original memory relies on the light and context A. Reexposure to the light in a very different context $\mathrm{B}$ allowed the second-order association of the shock with context B. This manipulation involved a consolidation process in the HPC rather than updating of the original memory. This result may be explained by the low similarity between contexts A and B (Fig. 1, red curve). The current model predicts that reexposure to the light in a slightly modified context A should have resulted in memory updating.

Conversely, in the work of Lee (2010), rats were repeatedly exposed to a given context in which they finally received an immediate electric footshock. In this case, the original memory relies heavily on the context. The immediate shock association with the context involved a memory up- 
dating process in the HPC. Although the context had been extensively experienced, the reactivation of the original memory occurred in exactly the same context, thus allowing memory updating in accordance with the current model (Fig. 1, blue curve).

In summary, the paper by Winters and colleagues (2011) may profoundly impact our comprehension of HPC contribution to memory updating. Together with the aforementioned studies, the results of Winters and colleagues (2011) prompted the model proposed herein, which may apply to several types of memories. This model posits that the degree of similarity between the events present at recall and past learning experience should bidirectionally gate new learning or memory updating in the HPC. In this regard, the HPC has previously been reported to act as a comparator between current and past similar events in humans (Kumaran and Maguire, 2007). If this model helps to reconcile previous findings regarding memory updating upon recall, an important question remains to be elucidated: Are new learning and memory updating truly dissociated in the HPC as stated in the present model, or are there circumstances under which the two processes coexist and perhaps interact? Future studies will undoubtedly shed new light on this question.

\section{References}

Bozon B, Davis S, Laroche S (2003) A requirement for the immediate early gene zif 268 in reconsolidation of recognition memory after retrieval. Neuron 40:695-701.

Davis S, Renaudineau S, Poirier R, Poucet B, Save E, Laroche S (2010) The formation and stability of recognition memory: what happens upon recall? Front Behav Neurosci 4:177.

Ennaceur A, Delacour J (1988) A new one-trial test for neurobiological studies of memory in rats. 1 . Behavioral data. Behav Brain Res 31:47-59.

Kumaran D, Maguire EA (2007) Match mismatch processes underlie human hippocampal responses to associative novelty. J Neurosci 27:8517-8524.

Lee JL (2009) Reconsolidation: maintaining memory relevance. Trends Neurosci 32:413-420.

Lee JL (2010) Memory reconsolidation mediates the updating of hippocampal memory content. Front Behav Neurosci 4:168.
McGaugh JL (1966) Time-dependent processes in memory storage. Science 153:1351-1358.

Nader K, Hardt O (2009) A single standard for memory: the case for reconsolidation. Nat Rev Neurosci 10:224-234.

Nader K, Schafe GE, Le Doux JE (2000) Fear memories require protein synthesis in the amygdala for reconsolidation after retrieval. Nature 406:722-726.

Rossato JI, Bevilaqua LR, Myskiw JC, Medina JH, Izquierdo I, Cammarota M (2007) On the role of hippocampal protein synthesis in the consolidation and reconsolidation of object recognition memory. Learn Mem 14:36-46.

Tronel S, Milekic MH, Alberini CM (2005) Linking new information to a reactivated memory requires consolidation and not reconsolidation mechanisms. PLoS Biol 3:e293.

Winters BD, Forwood SE, Cowell RA, Saksida LM, Bussey TJ (2004) Double dissociation between the effects of peri-postrhinal cortex and hippocampal lesions on tests of object recognition and spatial memory: heterogeneity of function within the temporal lobe. J Neurosci 24:5901-5908.

Winters BD, Tucci MC, Jacklin DL, Reid JM, Newsome J (2011) On the dynamic nature of the engram: evidence for circuit-level reorganization of object memory traces following reactivation. J Neurosci 31:17719-17728. 